\title{
BG04
}

\section{Fracture Interpretation in OBM}

\section{J. Dong* (Schlumberger), X.W. Sun (PetroChina Tarim Oilfield), Y. Xin (PetroChina Tarim Oilfield), Y.G. Tang (PetroChina Tarim Oilfield) \& Q.K. Wu (PetroChina Tarim Oilfield)}

\section{SUMMARY}

This study consists of detailed analysis of 17 high-definition microresistivity images from a tight gas reservoir in western China. Most of the images are acquired in OBM with very adverse conditions for the imager tool resulting in limited image quality. Through extensive comparison between core and images and between OBM images and WBM images, we have improved and evaluated the accuracy of natural fracture interpretation from OBM images. In particular, one well is drilled and logged in OBM and then logged again in WBM over the same interval to compare the fracture interpretation results. The comparison shows that even in very harsh logging conditions, most of the fractures interpreted from WBM images can also be identified on OBM images. Interestingly, open fractures on OBM images have a conductive colour likely because of the magnetite used as weighting material in the mud. Thus, zones of effective fractures can be identified. This possibility is validated by mud loss data. 


\section{Introduction}

It is critical to log microresistivity borehole images for continuous and oriented natural fracture data to understand gas production mechanisms in naturally fractured tight gas reservoirs. However, the widely used oil-base mud (OBM) in this type of reservoir has hindered acquisition of this critical data because of the limited image quality. In western China, high-temperature/high pressure (HT/HP) wells are drilled with 6.625-in bits in OBM of high oil-water ratio $(>80 / 20)$ and high mud weight $\left(>1.85 \mathrm{~g} / \mathrm{cm}^{3}\right)$. Together with a low average formation resistivity $(\sim 30 \mathrm{ohmm})$, as expected, such a challenging environment limits the image quality that an advanced high-definition microresistivity imager provides (Laronga et al., 2011). However, this case study demonstrates the possibility of accurately characterizing natural fractures with the tool in such adverse conditions.

\section{Methodology}

The mud and hole conditions and the formation resistivity, as described above, fall into the poorest range for the advanced high-definition microresistivity imager to operate in, and the quality of acquired images could only be rated as level 1 and 2 according to the criteria described by Laronga et al. (2011). Natural fractures are visible, but could be easily confused with other features (e.g., crossbedds and hole rugosity). Therefore, the fundamental issues for studying natural fractures in such an environment are how to improve the interpretation accuracy and how to quantitatively evaluate the interpretation results. In this study, the following procedures were carried out to solve these issues:

- Core and core scan photographic observation. Dip angles of fractures and crossbeds were measured to distinguish these two features. Fracture-cementing minerals were identified to confirm the electrical property of the fractures on images. Images and core scan photographs were compared to get a glimpse of disturbing factors such as bit marks and threading.

- Comparison between images acquired in OBM and water-base mud (WBM) from the same well. One well was drilled and logged in OBM. WBM was then used to replace the OBM, and the image was acquired again over the target zone to compare the image quality in different mud systems. The interpretation results from the two images were quantitatively compared.

- Fracture interpretation comparison across wells. Statistics and comparison of fracture density interpreted from 10 OBM images and 7 WBM images were made across the anticline in which both OBM and WBM wells are uniformly located. Mud loss data was used to identify zones of effective (open) fractures. Fracture electrical property was correlated with mud loss.

\section{Results}

High-angle dips, peaking at $70^{\circ}$ or higher, are observed for fractures on cores and core scan photographs, making it easier to distinguish fractures from crossbedds and bed boundaries that have dip angles lower than $40^{\circ}$. Most of fractures have good consistency in strike in a certain interval of the core. Bit marks and threading on cores are clearly identified on the images, leading to negligible ambiguities. A large proportion of fractures are cemented or partially cemented with calcite or anhydrite. Almost none are clay cemented. The electric property of the fractures on OBM images are expected to be all resistive as both the cementing minerals and the mud are resistive. Nonetheless, a large number of dark fractures are observed on OBM images. The most reasonable speculation about this elusive phenomenon is that the iron ore powder used as a weighting material and which consists of a high content of conductive magnetite improves the conductivity of the mud. The magnetite particles precipitate in the open fractures and result in conductive colours. Many drilling-induced and drilling-enhanced fractures also appear to be dark on OBM images.

As shown in Fig. 1a, about $79 \%$ of the fractures interpreted from the WBM image can also be identified from the OBM image. Fracture density curves have similar highs and lows. Compared to the WBM images (Figs $1 \mathrm{~b}$ and 1c), fractures on the OBM image are mostly not complete yet can easily be 


\section{EAGE}

fit by sinusoids. In the lower zone where most fractures on the WBM image are conductive, fractures on the OBM image are mostly dark coloured (dark blue tadpoles in Fig. 1a).

The average fracture density calculated based on 10 OBM images equals approximately $77 \%$ of that derived from $7 \mathrm{WBM}$ images acquired in the same anticline. Most of the wells exhibit the same trend, as shown in Fig. 1a; cemented (WBM) and bright (OBM) fractures are present in the upper part of the target zone whereas open (WBM) and dark (OBM) fractures are found in the lower part.. This is consistent with the fact that most of mud loss happens in the lower zones and, therefore, demonstrates the possibility of identifying effective fracture zones with OBM images.

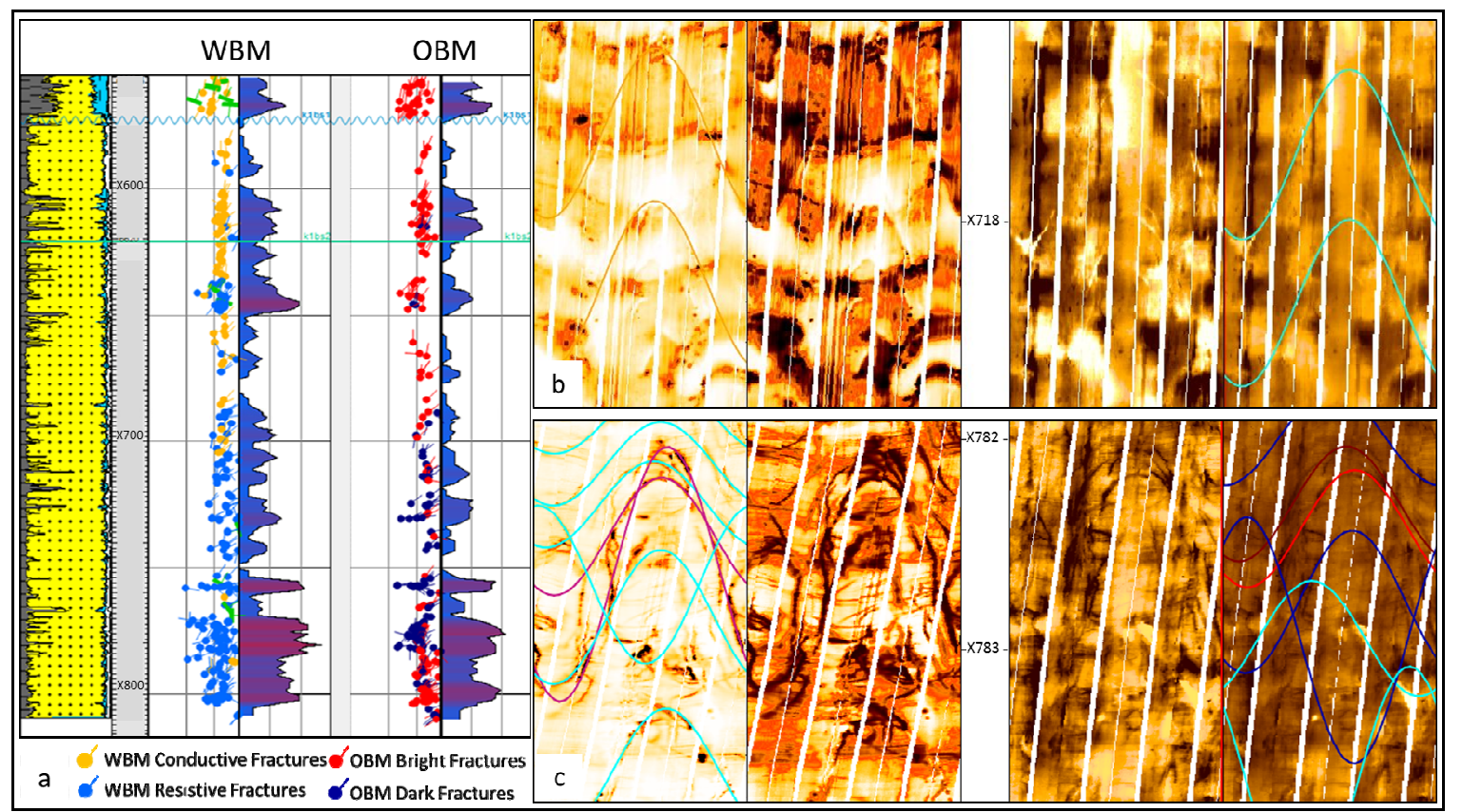

Figure 1 Comparison between images acquired in OBM and WBM from the same well. a) Interpreted fracture tadpoles and fracture densities. b) Resistive fractures on WBM image (left) and bright fractures on OBM image (right) in the same interval. c) Conductive fractures on WBM image (left) and dark fractures on OBM image (right) in the same interval.

\section{Conclusions}

This case study exemplifies the possibility of depicting natural fractures with an advanced highdefinition microresistivity imager in very harsh environments that are normally considered to be not feasible for the tool. This is particularly true if the ability of fracture capturing rather than image quality is the top priority for the geological application. Probably because of the magnetite as a weighting material in the mud, open fractures on OBM images have a dark colour. As a result, effective fracture zones can be identified.

\section{Acknowledgement}

The authors would like to thank PetroChina for use of the data.

\section{Reference}

Laronga, R., Lozada, G.T., Perez, F.M., Cheung, P., Hansen, S. M. and Rosas, A. M. [2011] A highdefinition approach to formation imaging in wells drilled with nonconductive muds. SPWLA $52^{\text {nd }}$ Annual Logging Symposium, 14-18 May. 